\title{
MicroRNA-372 functions as a tumor suppressor in cell invasion, migration and epithelial-mesenchymal transition by targeting $A T A D 2$ in renal cell carcinoma
}

\author{
SHIQI JI ${ }^{1 *}$, XIAOLIN SU ${ }^{2 *}$, HAIJIAN ZHANG ${ }^{1}$, ZHIXING HAN ${ }^{1}$, YUQIAN ZHAO ${ }^{1}$ and QINGJUN LIU ${ }^{1}$ \\ ${ }^{1}$ Department of Urology, Beijing Ditan Hospital Capital Medical University, Capital Medical University, Beijing 100015; \\ ${ }^{2}$ Department of Emergency, Beijing First Hospital of Integrated Chinese and Western Medicine, Beijing 100021, P.R. China
}

Received May 7, 2018; Accepted December 5, 2018

DOI: $10.3892 / \mathrm{ol} .2018 .9871$

\begin{abstract}
In recent years, renal cell carcinoma (RCC) has exhibited an increasing incidence and mortality rate worldwide. Accumulating evidence has identified that microRNAs (miRNAs) function as negative or positive regulators of many malignant tumors; however, the roles of miR-372 in RCC remain unclear. The focus of the present study was the functions of miR-372 in RCC metastasis and EMT. Data revealed that miR-372 expression levels were significantly downregulated in RCC tissue samples and cells. Moreover, the decreased expression levels were strongly associated with the poor survival rates and adverse clinical characteristics of RCC patients. Accordingly, miR-372 overexpression markedly inhibited RCC cell invasion, migration and EMT. In terms of the potential mechanisms, ATAD2, the expression of which was inversely correlated with miR-372 expression in RCC, was identified as a direct functional target of miR-372. Notably, ATAD2 silence exerted suppressive functions in RCC cells, being similar to the effects of miR-372 overexpression. In conclusion, findings of this study indicate that miR-372 repressed RCC EMT and metastasis via targeting ATAD2, suggesting that the miR-372/ $A T A D 2$ axis may be therapeutic biomarkers for RCC.
\end{abstract}

\section{Introduction}

Renal cell carcinoma (RCC), accounting for approximately $3 \%$ of human malignant tumors, is a common type of adult kidney tumor (1). Globally, over 260,000 patients are diagnosed with kidney tumor annually and over 116,000 patients succumb to the disease (2). The 5-year survival rate for RCC patients in

Correspondence to: Dr Qingjun Liu, Department of Urology, Beijing Ditan Hospital Capital Medical University, Capital Medical University, 8 Jingshun East Street, Chaoyang, Beijing 100015, P.R. China

E-mail: zuf974444@163.com

${ }^{*}$ Contributed equally

Key words: renal cell carcinoma, miR-372, migration, invasion, epithelial-mesenchymal transition, ATAD2 advanced stages remains poor due to metastasis or recurrence (3). Generally, the standard therapies for RCC involve nephron sparing surgery and radical nephrectomy $(4,5)$. However, these types of therapies are not expected to have curative effects as they only slightly extend progression-free survival (6). Therefore, further understanding of the molecular mechanisms underlying RCC metastasis and progression is of great importance to identify novel prognostic and diagnostic biomarkers for RCC.

miRNAs are a class of conserved non-coding RNAs, serving important roles in regulating gene expression levels via directly targeting the mRNA 3'-UTR to induce translation inhibition or mRNA degradation $(7,8)$. miRNAs have been confirmed to be involved in different kinds of cellular processes, such as apoptosis, metabolism, and differentiation (9-11). The study of miRNAs has provided a new direction in our understanding of the tumorigenic mechanism of RCC. Additionally, aberrant expression levels of various miRNAs have been observed in RCC. For instance, Song et al reported that miR-384 could repress RCC cell invasion and growth via regulating astrocyte elevated gene 1 (12). Wang et al reported that migration and invasion abilities of RCC were suppressed by miR-182 through the regulation of IGFIR (13). Hu et al revealed that miR-138 repressed RCC cell invasion and proliferation by mediating SOX9 (14). Thus, miRNAs may be underlying diagnostic markers for RCC.

Epithelial-mesenchymal transition (EMT) is a pathological and physiological process where epithelial cells lose their polarity and cell-cell adhesion signature, acquiring the mesenchymal characteristics $(15,16)$. During this progress, the expression levels of epithelial markers, such as E-cadherin, increase, while the expression levels of mesenchymal markers, such as vimentin, decrease (17). EMT plays a critical role in many aspects of tumor behavior, including metastasis (18). Thus, further investigation is essential to better understand the mechanisms underlying EMT progression in RCC.

ATAD2 (ATPase family AAA domain-containing protein 2), also known as $\mathrm{AAA}^{+}$nuclear coregulator cancer associated (ANCCA), is a member of the $\mathrm{AAA}^{+}$ATPase family (19). ATAD2 contains both an ATPase domain and a bromodomain. Additionally, ATAD2 maps to chromosome 8q24, which is the most commonly amplified region in multiple tumors $(20,21)$. The special structures of ATAD2 indicated that it was implicated 
in the regulation of genome, including cell differentiation, apoptosis, division and proliferation $(22,23)$. Recently, dysregulation of $A T A D 2$ has been found in various tumors, playing significant roles in tumor progression. For example, ATAD2 overexpression was associated with prognosis and progression in colorectal cancer (24); ATAD2 overexpression in gastric cancer served as a poor prognostic biomarker (25). Thus, studies have revealed that $A T A D 2$ manifests oncogenic functions in various malignancies. However, the significance of $A T A D 2$ in RCC remains uncertain.

\section{Materials and methods}

RCC clinical tissue specimens. Fifty-two pairs of RCC tissue specimens and matched non-cancerous tissues were collected from RCC patients who underwent radical nephrectomy in Beijing Ditan Hospital Capital Medical University (Beijing, China) between September 2015 and June 2017. None of the patients had received chemotherapy or radiotherapy prior to the surgery. All the tissues were immediately frozen in liquid nitrogen after resection and stored at $-80^{\circ} \mathrm{C}$ for further assays.

Written consent was obtained from the RCC patients involved in the current study. The study was approved by the Ethics Committees of Beijing Ditan Hospital Capital Medical University.

Cell lines and cell culture. Five human RCC cell lines (Caki-1, 786-O, A498, ACHN and Caki-2) and the immortalized normal human renal proximal tubule epithelial cell line HK-2 were obtained from the American Type Culture Collection (Manassas, VA, USA). All the RCC cells were maintained in RPMI-1640 medium (Gibco; Thermo Fisher Scientific, Inc., Waltham, MA, USA) while HK-2 was cultured in keratinocyte serum-free medium (Gibco; Thermo Fisher Scientific, Inc.). Both culture media contained 10\% FBS (Gibco; Thermo Fisher Scientific, Inc.), $100 \mathrm{U} / \mathrm{ml}$ penicillin and $100 \mu \mathrm{g} / \mathrm{ml}$ streptomycin (Corning Incorporated, Corning, NY, USA). All the cells were kept in a humidified incubator containing $5 \% \mathrm{CO}_{2}$ at $37^{\circ} \mathrm{C}$.

Cell transfection. The miR-372 mimics, inhibitors, ATAD2 overexpression plasmids and ATAD2 siRNA were produced by GenePharmaCo. (Shanghai, China) and transfected into RCC cells using Lipofectamine ${ }^{\circledR} 2000$ (Invitrogen; Thermo Fisher Scientific, Inc.) in line with the manufacturer's protocol. The cells were harvested $48 \mathrm{~h}$ post-transfection for further assays.

RT-qPCR. TRIzol (Invitrogen; Thermo Fisher Scientific, Inc.) was applied to isolate the total RNAs from RCC tissues and cultured cells following the manufacturers' protocol. Reverse transcription for cDNA was performed using the TaqMan MicroRNA Reverse Transcription kit (Applied Biosystems; Thermo Fisher Scientific, Inc.). Then, cDNA was amplified using a SYBR-Green mix kit and the ABI 7900 Real-Time PCR system (Applied Biosystems). Primer sequences are shown in Table I. GAPDH was used as endogenous controls for ATAD2 and EMT-related genes. U6 served as the internal control for miR-372. The relative expression levels were determined using the $2^{-\Delta \Delta C q}$ method (26). RT-qPCR was performed with the following thermocycling conditions: Initial denaturation at $95^{\circ} \mathrm{C}$ for $10 \mathrm{~min}$, followed by 40 cycles of $95^{\circ} \mathrm{C}$ for $15 \mathrm{sec}$, annealing at $50^{\circ} \mathrm{C}$ for $30 \mathrm{sec}$, and extension at $72^{\circ} \mathrm{C}$ for $30 \mathrm{sec}$.
Table I. Primer sequences for RT-qPCR.

\begin{tabular}{ll}
\hline Primer & \multicolumn{1}{c}{ Sequence } \\
\hline miR-372 & F: 5'-AGCCTAAAGTGCTGCGACATT-3' \\
& R: 5'-GTGCAGGGTCCGAGGT-3' \\
U6 & F: 5'-CTCGCTTCGGCAGCACA-3' \\
& R: 5'-AACGCTTCACGAATTTGCGT-3' \\
ATAD2 & F: 5'-GGAATCCCAAACCACTGGACA-3' \\
& R: 5'-GGTAGCGTCGTCGTAAAGCACA-3' \\
GAPDH & F: 5'-GAAGGTGAAGGTCGGAG-3' \\
& R: 5'-GAAGATGGTGATGGGAT-3' \\
E-cadherin & F: 5'-CACCTGGAGAGAGGCCATGT-3' \\
& R: 5'-TGGGAAACATGAGCAGCTCT-3'
\end{tabular}

Vimentin F: 5'-AGCTGCAGGCTCAGATTCAGGA-3' R: 5'-CGGTTGGCAGCCTCAGAGAGGT-3'

F, forward; R, reverse; U6, small nuclear RNA, snRNA; ATAD2, ATPase family AAA domain-containing protein 2; GAPDH, glyceraldehyde-3-phosphate dehydrogenase.

Transwell assays. Transwell chamber $(8.0 \mu \mathrm{m}$ pore size, Corning Incorporated) was applied to determine the RCC cell invasion and migration abilities with or without the Matrigel (Corning Incorporated) being precoated. For invasion assay, $1 \times 10^{4} \mathrm{RCC}$ cells in serum-free RPMI-1640 medium were placed into top chambers of the inserts which had been precoated with Matrigel. Then, culture medium containing $10 \%$ FBS, as a chemoattractant, was added into bottom chambers. After incubation at $37^{\circ} \mathrm{C}$ for $48 \mathrm{~h}$ in a $5 \% \mathrm{CO}_{2}$ atmosphere, the cells that remained on the top side of the inserts were removed using a cotton swab while the cells that invaded the bottom side were fixed with $0.1 \%$ paraformaldehyde for $15 \mathrm{~min}$ and stained with $0.1 \%$ crystal violet for $15 \mathrm{~min}$ at room temperature. Then, the stained cells were counted under a microscope (IX53; Olympus Corporation, Tokyo, Japan) from five randomly selected visual fields. The difference between the migration and invasion assays was that the insert was not plated with Matrigel for the migration assays.

Western blotting. Cultured cells were harvested and lysed with RIPA buffer (Beyotime, Shanghai, China) containing the protease inhibitors (Roche, Basel, Switzerland) on ice for 15 min. The BCA method (BCA protein assay kit; Beyotime) was used to determine the concentration of the proteins. Proteins $(40 \mu \mathrm{g})$ were then separated by $10 \%$ SDS-PAGE and transferred onto PVDF membranes (Millipore, Bedford, MA, USA). The membranes were blocked with $5 \%$ non-fat milk in TBST at room temperature for $2 \mathrm{~h}$, followed by incubation overnight at $4{ }^{\circ} \mathrm{C}$ with appropriate primary antibodies which were as follows: antibodies against ATAD2 (1:1000, ab118664, Abcam, Cambridge, MA, USA), Vimentin (1:1000, ab92547, Abcam), E-cadherin (1:1000, ab15148, Abcam) and GAPDH (1:1000, ab9485, Abcam). After incubation with the corresponding horseradish peroxidase-conjugated secondary antibodies (1:2000, ab6721, Abcam) for $2 \mathrm{~h}$ at room temperature, the protein bands were visualized using a chemiluminescent imaging system (Pierce, Rockford, IL, USA). Densitometry 


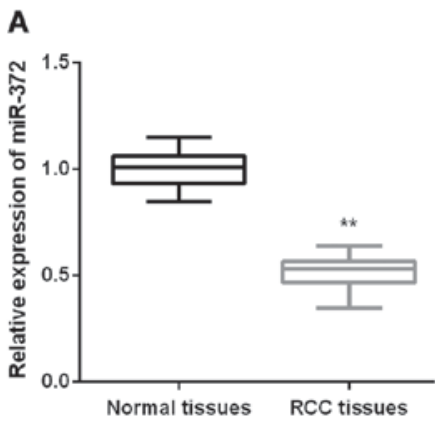

D

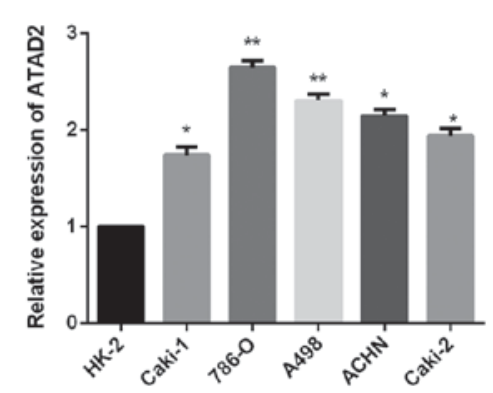

B

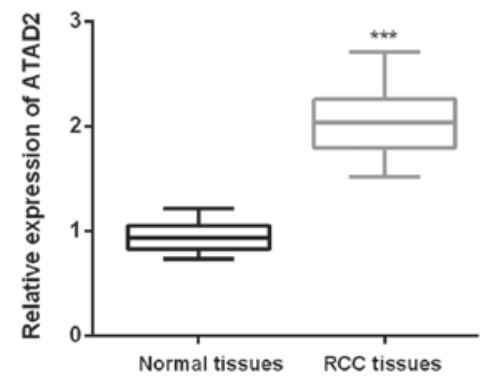

C

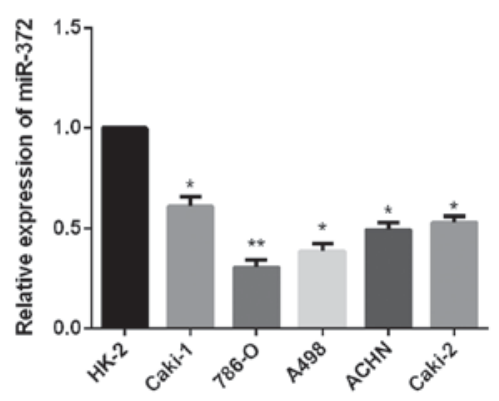

E

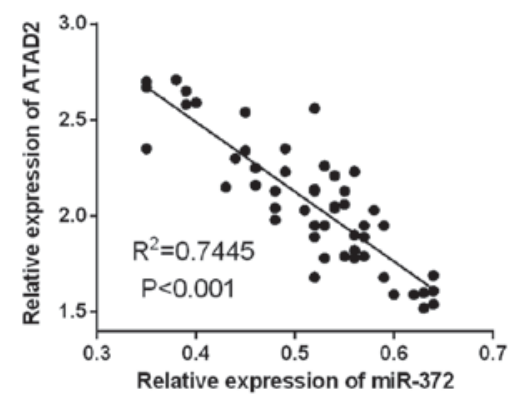

Figure 1. miR-372 is downregulated and ATAD2 is upregulated in RCC. (A) miR-372 expression levels in normal and RCC tissues. (B) ATAD2 expression levels in RCC tissues and adjacent normal tissues were detected by RT-qPCR. (C) miR-372 expression levels in RCC and normal renal cells. (D) ATAD2 expression levels in RCC and normal renal cells. (E) Spearman's correlation analysis between the miR-372 and ATAD2 expression levels in RCC tissues. "P<0.05, ${ }^{* * *} \mathrm{P}<0.01$, ${ }^{* * *} \mathrm{P}<0.001$

analysis of the protein blots was conducted using Image-Pro Plus 6.0 software (Media Cybernetics, Inc., Rockville, MD, USA). GAPDH was used as an internal control.

Luciferase reporter assays. The wild-type (WT) or mutant (MUT) ATAD2-3'UTR which contained the miR-372 binding sites was inserted into the luciferase genes in the pGL3 vectors (Promega, Madison, WI, USA). RCC cells were co-transfected with pGL3-WT ATAD2-3'-UTR or pGL3-MUT ATAD23'-UTR and miR-372 mimics. The luciferase activities were detected with the dual-luciferase reporter assay kit (Promega) $48 \mathrm{~h}$ after the transfections.

Statistical analysis. Statistical analyses were performed using SPSS software version 17.0 (SPSS Inc., Chicago, IL, USA). Student's t-test, ANOVA and Scheffe post-hoc analysis were applied, where appropriate. A paired Student's t-test was performed to analyze the paired data. For analysis on clinical correlation between miR-372 expression and RCC patients' clinicopathological features, Pearson's Chi-square test was used. Spearman's correlation method was applied to estimate the correlations between mRNA and miRNA. Kaplan-Meier method was applied to determine the survival rates, and the log-rank test was performed to compare the difference between the survival curves. $\mathrm{P}<0.05$ was considered to be statistically significant difference.

\section{Results}

Aberrant expression levels of $m i R-372$ and ATAD2 in RCC. To validate whether miR-372 functionally modulates ATAD2 expression levels in RCC, firstly, we measured miR-372 and $A T A D 2$ expression levels in RCC tissue samples. Results revealed that RCC tissues presented a lower miR-372 expression level in comparison with the non-tumor tissues (Fig. 1A), whereas, the expression levels of ATAD2 in RCC tissues were prominently upregulated when compared to the paired adjacent normal tissues (Fig. 1B). Similarly, significantly decreased miR-372 expression levels were detected in RCC cells as compared to the normal renal cell HK-2 (Fig. 1C). We further evaluated ATAD2 expression levels in RCC cells. As shown in Fig. 1D, ATAD2 expression levels were found to be markedly increased in RCC cells compared to HK-2. Additionally, the correlation between miR-372 and ATAD2 expression levels in RCC tissues was analyzed. The finding of Spearman's correlation rank test demonstrated a negative correlation between miR-372 and ATAD2 expression levels (Fig. 1E).

miR-372 suppresses RCC cell migration and invasion. To investigate the functional roles of miR-372 in RCC progression, we performed miR-372 inhibited or overexpressed assays in Caki-1 and 786-O cells, which had the relatively highest or lowest endogenous miR-372 expression levels, by transfecting the miR-372 mimics or inhibitor into $786-\mathrm{O}$ or Caki-1 cells. Successful overexpression or knockdown of miR-372 in 786-O or Caki-1 cells was confirmed using RT-qPCR (Fig. 2A and 2C). Then, Transwell assays were conducted to confirm the functions of miR-372 in RCC cell migration and invasion. The findings showed that miR-372 overexpression significantly reduced 786-O cell migration and invasion (Fig. 2B). By contrast, there was a significant increase in cell invasion and migration of Caki-1 treated with miR-372 inhibitor (Fig. 2D).

miR-372 suppresses RCC cell EMT. Next, we monitored alterations of EMT markers, including Vimentin and E-Cadherin, to investigate the functions of miR-372 in EMT of RCC cells. 
A

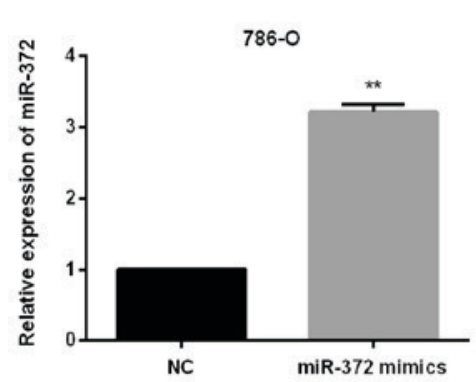

C

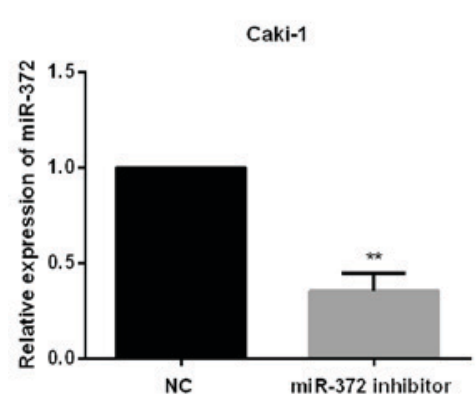

B
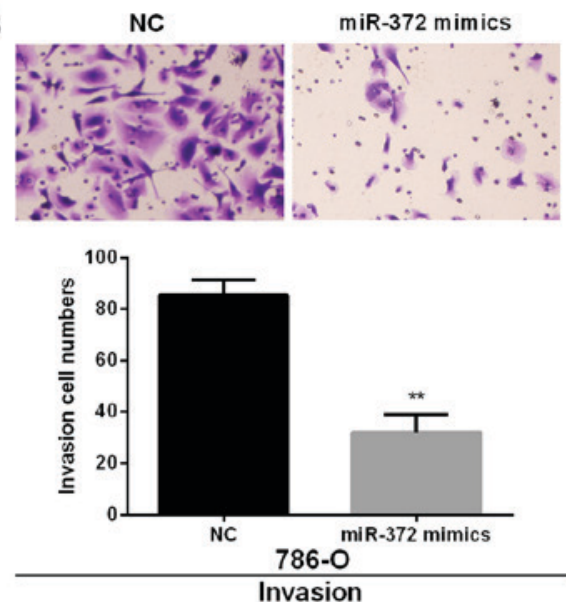

D

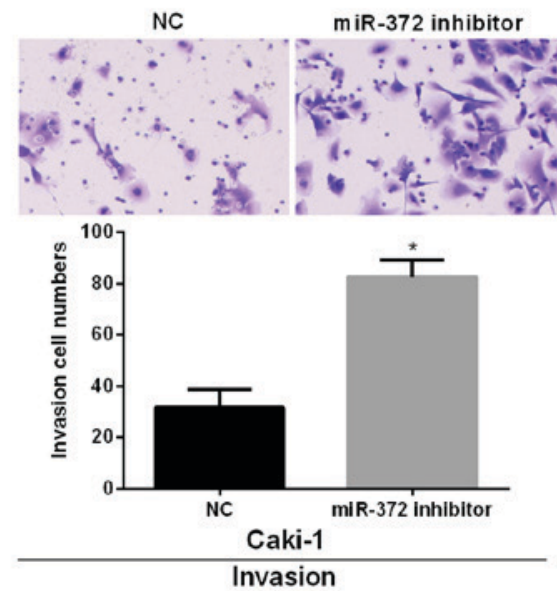

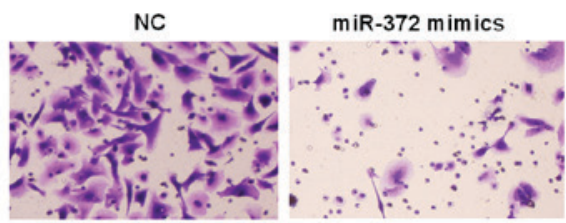

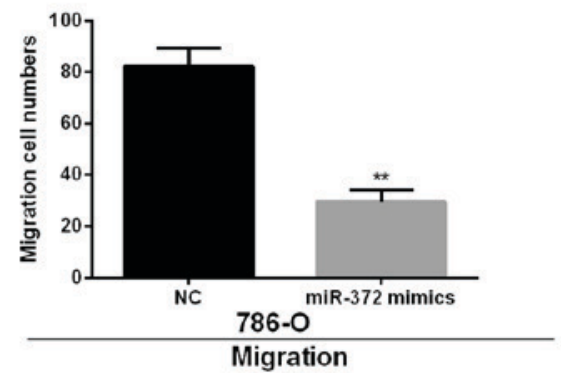

Migration

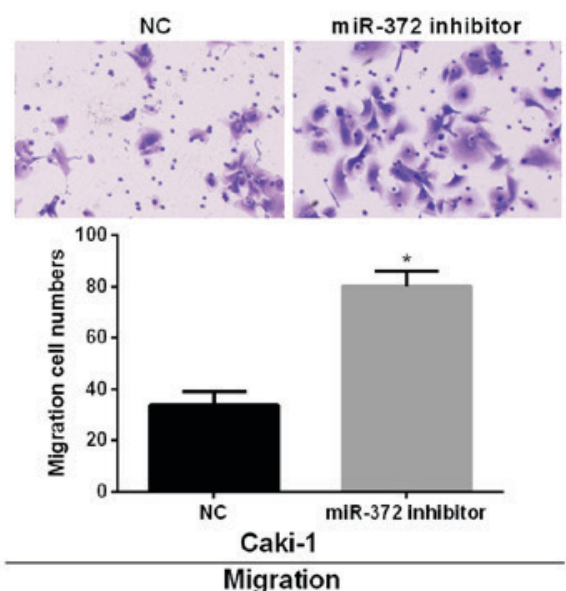

Figure 2. miR-372 suppresses cell invasion and migration in RCC cells. (A) miR-372 expression levels in 786-O cells with transfection of miR-372 mimics. (B) Cell invasion and migration were measured by Transwell assays in 786-O treated with miR-372 mimics. (C) miR-372 expression levels in Caki-1 cells with transfection of miR-372 inhibitor. (D) Cell invasion and migration were measured by Transwell assays in Caki-1 cells with transfection of miR-372 inhibitor. ${ }^{*} \mathrm{P}<0.05,{ }^{* *} \mathrm{P}<0.01$.

A

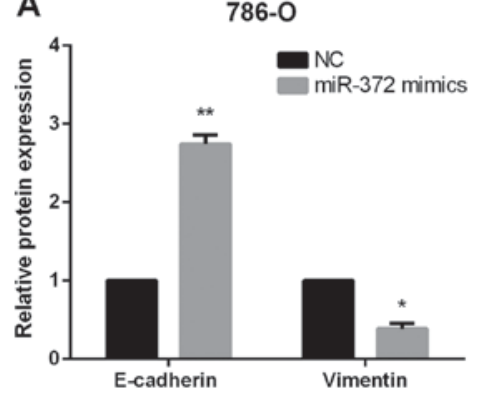

C

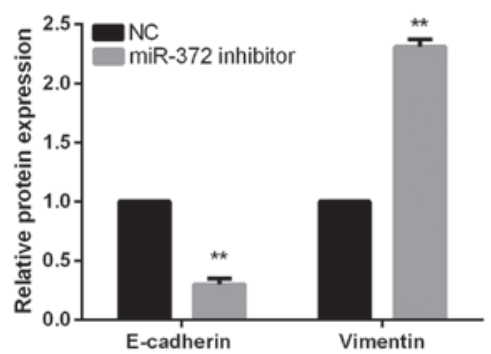

B

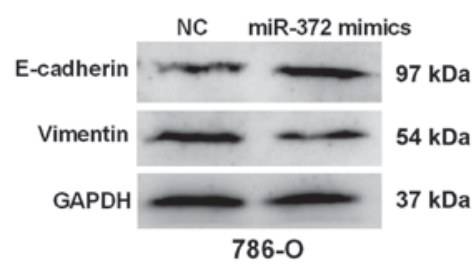

D

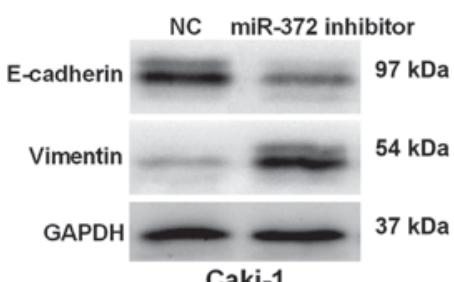

Caki-1

Figure 3. miR-372 suppressed EMT in RCC cells. (A and B) miR-372 overexpression in 786-O cells elevated the E-cadherin expression levels and reduced the Vimentin expression levels. (C and D) Inhibition of miR-372 in Caki-1 cells downregulated the E-cadherin expression levels and enhanced the Vimentin expression levels. ${ }^{*} \mathrm{P}<0.05,{ }^{* *} \mathrm{P}<0.01$. 
A

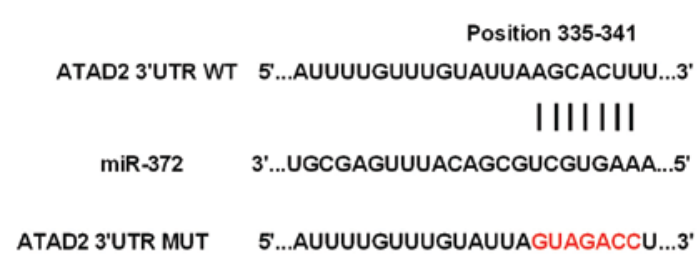

C

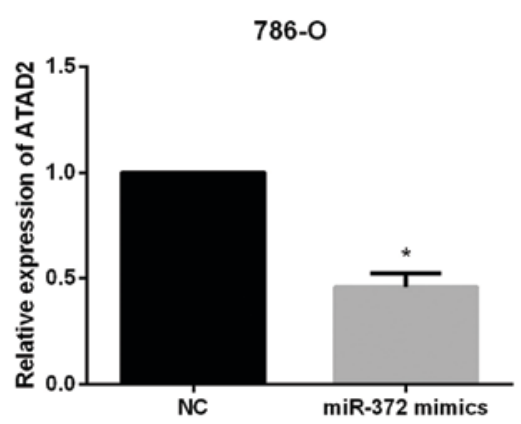

B

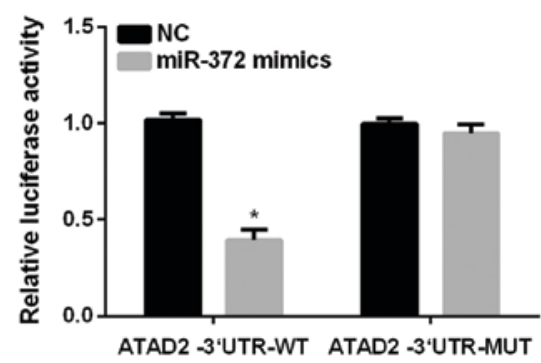

D

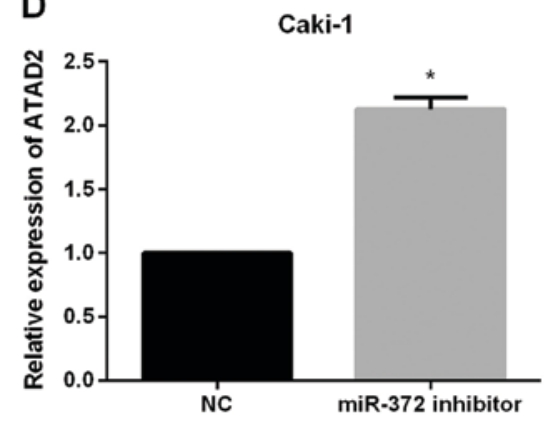

Figure 4. ATAD2 is the direct target of miR-372 in RCC. (A) The putative binding sites of miR-372 in the ATAD2 3'-UTR. (B) The luciferase activities of the ATAD2 3'UTR was detected by luciferase reporter gene assays in RCC cells which were cotransfected with wild-type ATAD2 3'UTR or mutational type ATAD2 3'UTR and miR-372 mimics, respectively. (C) ATAD2 expression in 786-O treated with miR-372 mimics. (D) ATAD2 expression in Caki-1 treated with miR-372 inhibitor. ${ }^{*} \mathrm{P}<0.05$.

As expected, results of RT-qPCR and western blot analysis both demonstrated that E-Cadherin expression levels were obviously increased, whereas the Vimentin expression levels were markedly reduced in miR-372 overexpressed 786-O cells (Fig. 3A and B). By contrast, Vimentin was upregulated in miR-372-suppressed Caki-1 cells, while E-Cadherin was prominently downregulated (Fig. $3 \mathrm{C}$ and D). Our data revealed that miR-372 overexpression could suppress the RCC cell EMT progression.

ATAD2 is a direct target of miR-372 in RCC cell lines. The significant impact of miR-372 on the metastasis of RCC cells prompted us to investigate the mechanisms underlying its functional effects. The data from Targetscan database revealed that $A T A D 2$ had complementary binding sites for miR-372 (Fig. 4A). Then, dual-luciferase reporter assays were performed to validate the association between ATAD2 and miR-372. The results showed that miR-372 significantly reduced the luciferase activities of ATAD2-3'UTR-WT without having obvious effects on that of ATAD2-3'UTR-Mut (Fig. 4B). We also investigated whether miR-372 could regulate the ATAD2 expression levels in RCC cells. The results of RT-qPCR indicated that miR-372 overexpression significantly reduced $A T A D 2$ expression levels in 786-O cells (Fig. 4C) while inhibition of miR-372 significantly elevated ATAD2 expression levels in Caki-1 cells (Fig. 4D).

ATAD2 mediated the biological functions of miR-372 in RCC cell invasion and migration. Next, we performed rescue experiments to verify that miR-372 inhibited RCC metastasis ability by directly reducing $A T A D 2$. First, we obtained miR-372 overexpressed 786-O cells or miR-372 suppressed Caki-1 cells by transfecting miR-372 mimics or inhibitor. Then, to verify that the biological functions of miR-372 in RCC was modulated by ATAD2, we transfected ATAD2 overexpressing vector or ATAD2 siRNA into miR-372 overexpressed 786-O cells or miR-372 suppressed Caki-1 cells, inducing an increase or decrease of ATAD2 expression (Fig. 5A and B). Next, Transwell assays were performed to determine the migration and invasion capacities. Findings demonstrated that ATAD2 upregulation markedly enhanced the invasion and migration abilities of miR-372 overexpressed 786-O cells (Fig. 5C). On the contrary, knockdown of ATAD2 dramatically reduced the invasion and migration abilities of miR-372 suppressed Caki-1 cells (Fig. 5D). miR-372 inhibited RCC cell EMT by regulating $A T A D 2$. We further investigated the functions of $A T A D 2$ in mediating the functional roles of miR-372 in RCC EMT progress by qRT-PCR and western blots. As expected, in miR-372 overexpressed 786-O cells, E-cadherin expression levels were significantly reduced and vimentin expression levels were prominently enhanced by $A T A D 2$ overexpression (Fig. 6A and 6B). However, ATAD2 silence significantly elevated the E-cadherin expression levels and decreased the vimentin expression levels in miR-372 suppressed Caki-1 cells (Fig. 6C and D). The data indicated that miR-372 suppressed RCC cell EMT process by regulation of ATAD2.

Aberrant expression levels of miR-372 and ATAD2 were correlated with clinicopathological features and prognosis of RCC patients. As we had confirmed the functional effects of miR-372 and ATAD2 in RCC, subsequently, we further investigated the clinical significance of miR-372 and ATAD2 in RCC. The RCC patients involved in current study were divided into two groups based on the mean expression level 
A

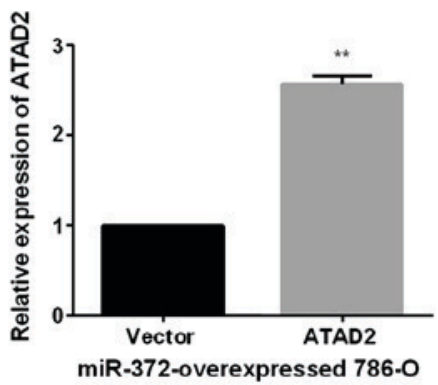

C
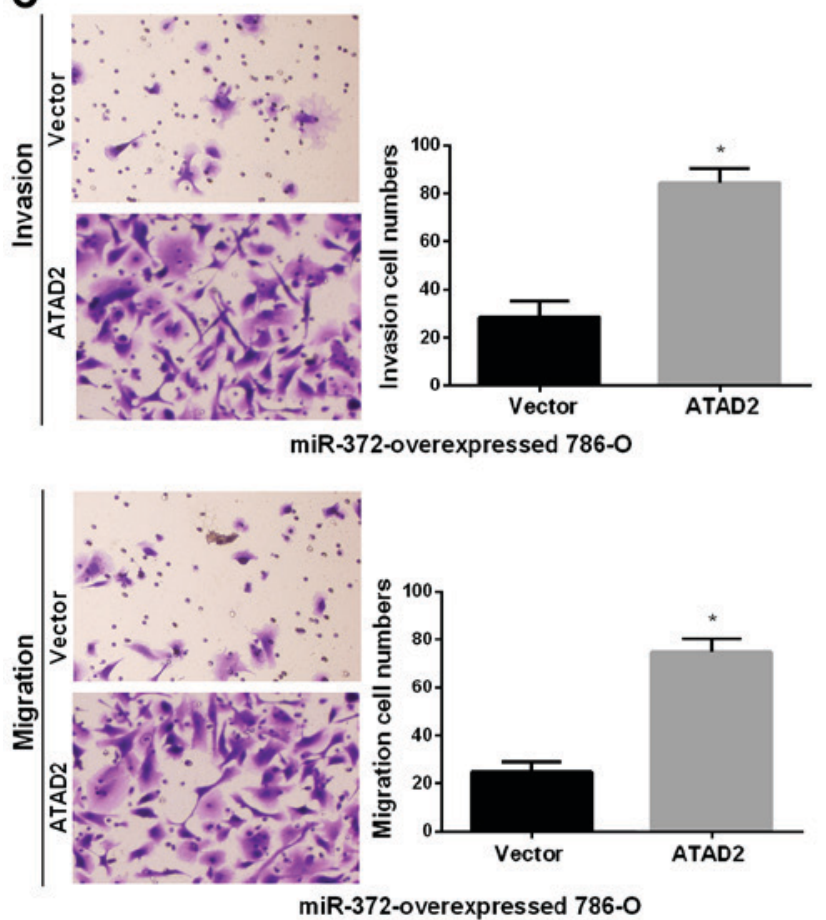

B

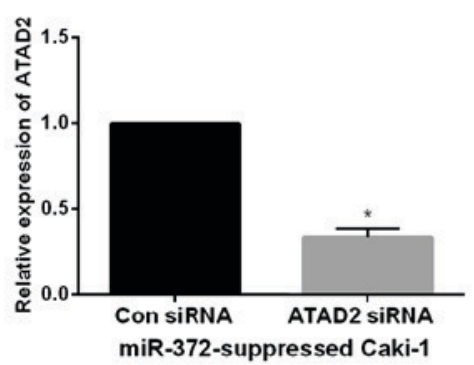

D
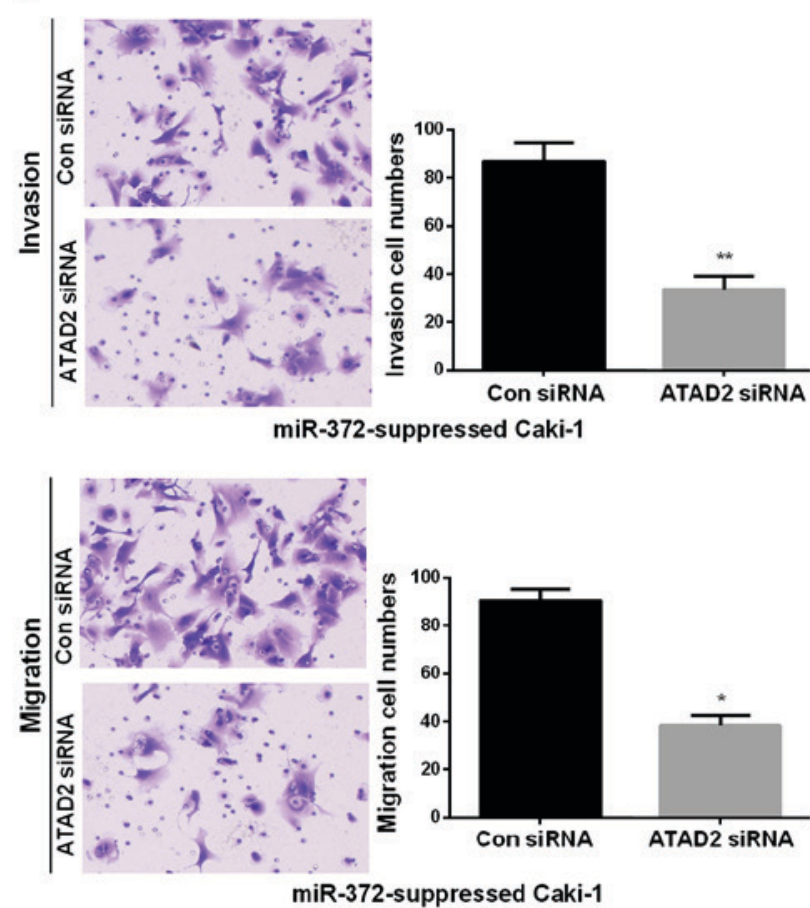

Figure 5. Altering expression of ATAD2 partly reverses miR-372-mediated functions in RCC cell invasion and migration. (A and B) ATAD2 expression levels in miR-372-overexpressed 786-O cells and miR-372-suppressed Caki-1 cells, which were cotransfected with ATAD2 overexpression plasmid or ATAD2 siRNA respectively. (C and D) Transwell assays were conducted to determine cell invasion and migration abilities of (C) miR-372-overexpressed 786-O cells and (D) miR-372-suppressed Caki-1 cells, which were cotransfected with $A T A D 2$ overexpression plasmid or ATAD2 siRNA respectively. ${ }^{*} \mathrm{P}<0.05,{ }^{* *} \mathrm{P}<0.01$.

of miR-372. Kaplan-Meier abalysis demonstrated that RCC patients with relatively low miR-372 expression levels had significantly decreased OS (Fig. 7A, P=0.0312) and DFS (Fig. 7B, $\mathrm{P}=0.0366$ ). In addition, ATAD2 high-expressing RCC patients presented shorter OS and DFS compared to ATAD2 low-expressing cases (Fig. 7C and D). Correlation between miR-372 expression and clinical features analysis implied that miR-372 expression levels were relevant to lymph node metastasis, TNM stage and tumor differentiation $(\mathrm{P}=0.0015$, 0.0075 and 0.0103 , respectively, Table II).

\section{Discussion}

RCC is the most common neoplasm of the adult kidney, and the morbidity and mortality rates are increasing at a rate of 2-3\% per decade (27). However, the curative effects of radiotherapy and traditional chemotherapy in advanced RCC therapy are limited (28). Additionally, although surgery is often curative for localized disease, majority of these patients experience metastasis or relapses, which is associated with poor prognosis. Therefore, it is of crucial significance to understand the mechanisms underlying RCC metastasis and recurrence to explore novel anti-RCC therapeutic targets. Previous studies revealed that miRNAs may provide new directions for earlier diagnosis as well as better prognosis of RCC (29).

Recent studies have proposed the antitumor effects of miR-372 in various malignancies. Aberrant miR-372 expression has been identified as novel prognostic as well as diagnostic marker for osteosarcoma (30) and colorectal cancer (31). Additionally, studies by $\mathrm{Wu}$ et al indicated that declined miR-372 expression in hepatocellular carcinoma was relevant to tumor metastasis and poor prognosis (32); Kong et al reported that miR-372 repressed prostate cancer cell invasion and migration via regulating $p 65$ (33); Liu et al found that miR-372 inhibited the progression of endometrial carcinoma via the regulation of $R h o C$ (34). Consistent with these findings, we found that miR-372 was obviously downregulated in RCC and its decreased expression levels were related to poor prognosis and worse clinicopathological parameters of RCC patients. Furthermore, miR-372 overexpression suppressed RCC cell EMT and metastasis. The data suggested that miR-372 played an inhibitory role in RCC metastasis and progression. 
A

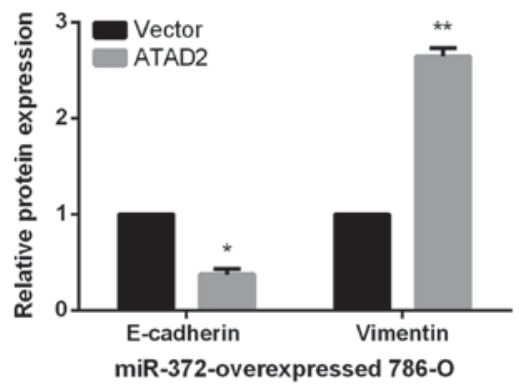

C

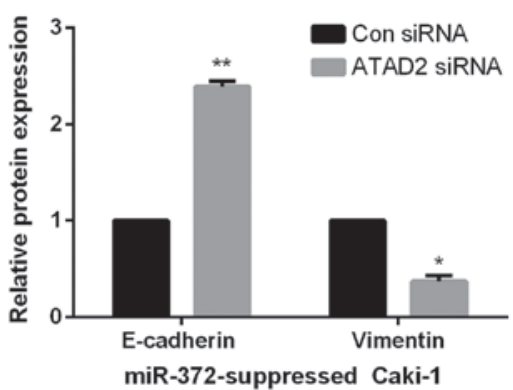

B

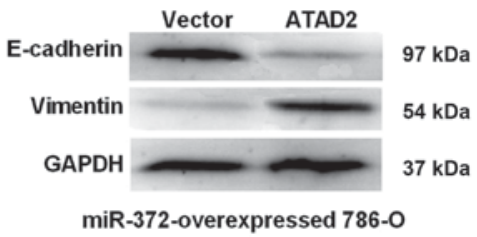

D

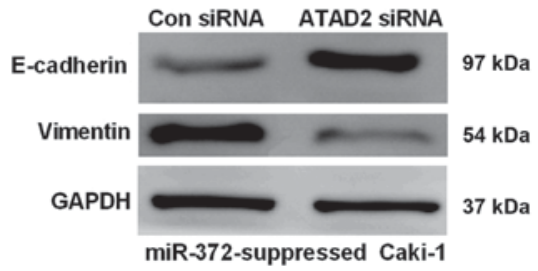

Figure 6. Altering of ATAD2 expression levels reversed the effect of miR-372 on RCC cell EMT process. (A and B) Overexpression of ATAD2 in miR-372-overexpressed 786-O cells decreased the E-cadherin expression levels and increased the Vimentin expression levels. (C and D) Knockdown of ATAD2 in miR-372-suppressed Caki-1 cells upregulated E-cadherin and downregulated Vimentin significantly. ${ }^{*} \mathrm{P}<0.05,{ }^{* *} \mathrm{P}<0.01$.

A

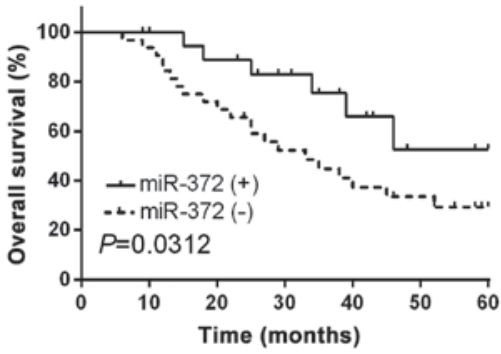

C

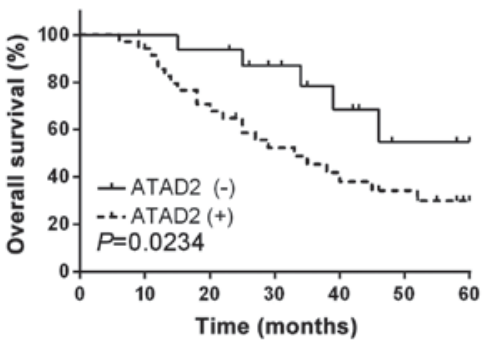

B

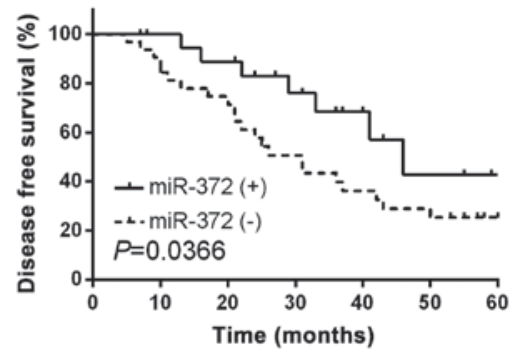

D

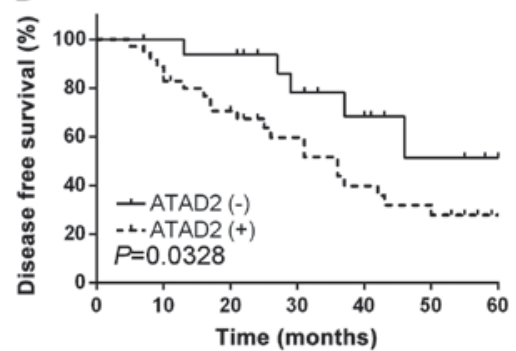

Figure 7. Prognostic value of miR-372 and ATAD2 in RCC patients. (A) OS and (B) DFS of RCC patients with high and low miR-372 expression levels. (C) OS and (D) DFS of RCC patients with high and low ATAD2 expression levels.

Previous studies have identified and validated an array of possible direct targets of miR-372, such as TXNIP, ULKI and $C D K 2$ (35-37), suggesting that miR-372 is an antitumor miRNA which plays a pivotal role in the initiation and progression of cancer. To extend that observation, we assessed the relationship between miR-372 and ATAD2 in RCC. ATAD2 has been identified as a novel candidate oncogene and possibly a therapeutic target for several types of human cancers (38). For instance, Hwang et al found that ATAD2 functioned as a poor prognostic biomarker in hepatocellular carcinoma (39);
Wan et al reported that ATAD2 overexpression in ovarian carcinoma indicated poor prognosis (40); Shang et al reported that overexpression of ATAD2/ANCCA in endometrial carcinoma was related to poor prognosis and tumor progression (41). These studies suggested that ATAD2 might have a potential as a therapeutic target for malignancies. Although the correlation between clinicopathological features and ATAD2 expression levels has been studied in multiple tumors, little is known about $A T A D 2$ in RCC. Herein, the ectopic upregulation of $A T A D 2$ in RCC was presented, which demonstrated a poor 
Table II. Correlation of miR-372 expression with the clinicopathological characteristics of the RCC patients.

\begin{tabular}{|c|c|c|c|c|c|}
\hline \multirow[b]{2}{*}{ Clinicopathological features } & \multirow[b]{2}{*}{ Cases $(n=52)$} & \multicolumn{2}{|c|}{ miR-372a expression } & \multirow[b]{2}{*}{ P-value } & \multirow[b]{2}{*}{$\chi^{2}$ value } \\
\hline & & High $(n=21)$ & Low $(\mathrm{n}=31)$ & & \\
\hline Age (years) & & & & 0.2016 & 0.3718 \\
\hline$>60$ & 27 & 11 & 16 & & \\
\hline$\leq 60$ & 25 & 10 & 15 & & \\
\hline Sex & & & & 0.3124 & 0.0132 \\
\hline Male & 26 & 9 & 17 & & \\
\hline Female & 26 & 12 & 14 & & \\
\hline Tumor size (cm) & & & & 0.0614 & 2.1431 \\
\hline$\geq 5.0$ & 24 & 6 & 18 & & \\
\hline$<5.0$ & 28 & 15 & 13 & & \\
\hline TNM stage & & & & $0.0075^{\mathrm{b}}$ & 7.3920 \\
\hline I-II & 26 & 17 & 9 & & \\
\hline III & 26 & 4 & 22 & & \\
\hline Histological type & & & & 0.0765 & 1.0125 \\
\hline Clear cell & 28 & 12 & 16 & & \\
\hline Papillary & 24 & 9 & 15 & & \\
\hline Tumor differentiation & & & & $0.0103^{\mathrm{b}}$ & 3.7846 \\
\hline Well and Moderate & 25 & 16 & 9 & & \\
\hline Poor & 27 & 5 & 22 & & \\
\hline Lymph node metastasis & & & & $0.0015^{\mathrm{b}}$ & 9.5623 \\
\hline Present & 26 & 4 & 22 & & \\
\hline Absent & 26 & 17 & 9 & & \\
\hline
\end{tabular}

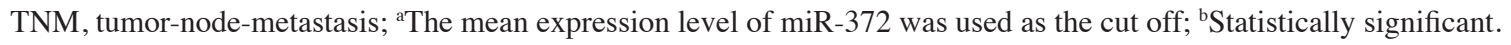

prognosis of RCC patients. Moreover, ATAD2 was identified as a direct target of miR-372 in RCC cells. Importantly, the inhibitory functions of miR-372 in RCC cell metastasis and EMT were confirmed to be regulated by ATAD2.

In conclusion, the findings in current research demonstrated a significant decrease of miR-372 expression levels and a notable increase of ATAD2 expression levels in RCC. Additionally, miR-372 overexpression had similar effects on repressing RCC cell invasion, migration and EMT as ATAD2 silence. Moreover, ATAD2 was confirmed to be a direct target of miR-372 and the antitumor effects of miR-372 on RCC progression were partially mediated by $A T A D 2$. The findings suggested that miR-372/ATAD2 axis may be attractive biomarkers and therapeutic targets for RCC patients.

\section{Acknowledgements}

Not applicable.

\section{Funding}

The study was supported by the Key Youth Project for Training Excellent Talents of Beijing (3101-03-36-10) and The Fundamental clinical cooperation project of Capital Medical University (17JL37).

\section{Availability of data and materials}

The datasets used and/or analyzed during the present study are available from the corresponding author on reasonable request.

\section{Authors' contributions}

SJ and XS contributed to the conception of the study; HZ contributed significantly to data analysis and manuscript preparation; $\mathrm{ZH}$ and $\mathrm{YZ}$ performed the data analyses and wrote the manuscript; QL performed the statistical analysis with constructive discussions. All authors read and approved the final manuscript.

\section{Ethics approval and consent to participate}

The study was approved by the Ethics Committee of Beijing Ditan Hospital Capital Medical University (Beijing, China). Signed informed consents were obtained from the patients or the guardians.

\section{Patient consent for publication}

Not applicable. 


\section{Competing interests}

The authors declare that they have no competing interests.

\section{References}

1. Siegel RL, Miller KD and Jemal A: Cancer Statistics, 2017. CA Cancer J Clin 67: 7-30, 2017.

2. Ridge CA, Pua BB and Madoff DC: Epidemiology and staging of renal cell carcinoma. Semin Intervent Radiol 31: 3-8, 2014.

3. Gupta K, Miller JD, Li JZ, Russell MW and Charbonneau C Epidemiologic and socioeconomic burden of metastatic renal cell carcinoma (mRCC): A literature review. Cancer Treat Rev 34: 193-205, 2008.

4. Jansi Prema KS, Devanathan KS and Kurien AA: Renal cell carcinoma with $\mathrm{t}(6,11)$ : A case report and review of literature. Indian J Pathol Microbiol 60: 574-576, 2017.

5. Gill DM, Hahn AW, Hale P and Maughan BL: Overview of current and future first-line systemic therapy for metastatic clear cell renal cell carcinoma. Curr Treat Options Oncol 19: 6, 2018.

6. Margulis V, Master VA, Cost NG, Leibovich BC, Joniau S Kuczyk M, Mulders PF, Kirkali Z, Wirth MP, Hirao Y, et al: International consultation on urologic diseases and the European Association of Urology international consultation on locally advanced renal cell carcinoma. Eur Urol 60: 673-683, 2011.

7. Bartel DP: MicroRNAs: Genomics, biogenesis, mechanism, and function. Cell 116: 281-297, 2004.

8. Lin S and Gregory RI: MicroRNA biogenesis pathways in cancer Nat Rev Cancer 15: 321-333, 2015

9. Cirilo PDR, de Sousa Andrade LN, Corrêa BRS, Qiao M, Furuya TK, Chammas R and Penalva LOF: MicroRNA-195 acts as an anti-proliferative miRNA in human melanoma cells by targeting Prohibitin 1. BMC Cancer 17: 750, 2017

10. Gang L, Qun L, Liu WD, Li YS, Xu YZ and Yuan DT: MicroRNA-34a promotes cell cycle arrest and apoptosis and suppresses cell adhesion by targeting DUSP1 in osteosarcoma. Am J Trans Res 9: 5388-5399, 2017.

11. Xu Y, Du J,Zhang P,Zhao X, Li Q, Jiang A, Jiang D, Tang G, Jiang Y Wang J, et al: MicroRNA-125a-5p mediates 3T3-L1 preadipocyte proliferation and differentiation. Molecules 23: E317, 2018.

12. Song H, Rao Y, Zhang G and Kong X: MicroRNA-384 inhibits the growth and invasion of renal cell carcinoma cells by targeting astrocyte elevated gene 1. Oncol Res: Aug 25, 2017 (Epub ahead of print).

13. Wang X, Li H, Cui L, Feng J and Fan Q: MicroRNA-182 suppresses clear cell renal cell carcinoma migration and invasion by targeting IGF1R. Neoplasma 63: 717-725, 2016.

14. Hu B, Wang J and Jin X: MicroRNA-138 suppresses cell proliferation and invasion of renal cell carcinoma by directly targeting SOX9. Oncol Lett 14: 7583-7588, 2017.

15. Chilosi M, Caliò A, Rossi A, Gilioli E, Pedica F, Montagna L, Pedron S, Confalonieri M, Doglioni C, Ziesche R, et al: Epithelia to mesenchymal transition-related proteins ZEB1, $\beta$-catenin, and $\beta$-tubulin-III in idiopathic pulmonary fibrosis. Mod Pathol 30: 26-38, 2017.

16. Cruz-Solbes AS and Youker K: Epithelial to mesenchymal transition (EMT) and endothelial to mesenchymal transition (EndMT): Role and implications in kidney fibrosis. Results Probl Cell Differ 60: 345-372, 2017.

17. Fedele M, Cerchia L and Chiappetta G: The epithelial-tomesenchymal transition in breast cancer: Focus on basal-like carcinomas. Cancers (Basel) 9: E134, 2017.

18. Saito N, Mine N, Kufe DW, Von Hoff DD and Kawabe T: CBP501 inhibits EGF-dependent cell migration, invasion and epithelialto-mesenchymal transition of non-small cell lung cancer cells by blocking KRas to calmodulin binding. Oncotarget 8 : 74006-74018, 2017.

19. Zou JX, Revenko AS, Li LB, Gemo AT and Chen HW: ANCCA, an estrogen-regulated $\mathrm{AAA}^{+}$ATPase coactivator for ERalpha, is required for coregulator occupancy and chromatin modification. Proc Natl Acad Sci USA 104: 18067-18072, 2007.

20. Beroukhim R, Mermel CH, Porter D, Wei G, Raychaudhuri S, Donovan J, Barretina J, Boehm JS, Dobson J, Urashima M, et al: The landscape of somatic copy-number alteration across human cancers. Nature 463: 899-905, 2010.

21. Raeder MB, Birkeland E, Trovik J, Krakstad C, Shehata S Schumacher S, Zack TI, Krohn A, Werner HM, Moody SE, et al Integrated genomic analysis of the 8q24 amplification in endometrial cancers identifies ATAD2 as essential to MYC-dependent cancers. PLoS One 8: e54873, 2013.
22. Zheng L, Li T, Zhang Y, Guo Y, Yao J, Dou L and Guo K: Oncogene ATAD2 promotes cell proliferation, invasion and migration in cervical cancer. Oncol Rep 33: 2337-2344, 2015.

23. Cattaneo M, Morozumi Y,Perazza D, Boussouar F, Jamshidikia M, Rousseaux S, Verdel A and Khochbin S: Lessons from yeast on emerging roles of the ATAD2 protein family in gene regulation and genome organization. Mol Cells 37: 851-856, 2014

24. Hou M, Huang R, Song Y, Feng D, Jiang Y and Liu M: ATAD2 overexpression is associated with progression and prognosis in colorectal cancer. Jpn J Clin Oncol 46: 222-227, 2016.

25. Zhang M, Zhang C, Du W, Yang X and Chen Z: ATAD2 is overexpressed in gastric cancer and serves as an independent poor prognostic biomarker. Clin Transl Oncol 18: 776-781, 2016.

26. Livak KJ and Schmittgen TD: Analysis of relative gene expression data using real-time quantitative PCR and the 2(-Delta Delta C(T)) method. Methods 25: 402-408, 2001.

27. Hakimi AA, Furberg H, Zabor EC, Jacobsen A, Schultz N, Ciriello G, Mikklineni N, Fiegoli B, Kim PH, Voss MH, et al: An epidemiologic and genomic investigation into the obesity paradox in renal cell carcinoma. J Natl Cancer Inst 105: 1862-1870, 2013.

28. Massari F, Di Nunno V, Ciccarese C, Graham J, Porta C, Comito F, Cubelli M, Iacovelli R and Heng DYC: Adjuvant therapy in renal cell carcinoma. Cancer Treat Rev 60: 152-157, 2017.

29. Zhu J, Ma X, Zhang Y, Ni D, Ai Q, Li H and Zhang X: Establishment of a miRNA-mRNA regulatory network in metastatic renal cell carcinoma and screening of potential therapeutic targets. Tumour Biol 37: 15649-15663, 2016.

30. Xu SY, Xu PF and Gao TT: MiR-372-3p inhibits the growth and metastasis of osteosarcoma cells by targeting FXYD6. Eur Rev Med Pharmacol Sci 22: 62-69, 2018

31. Yu J, Jin L, Jiang L, Gao L, Zhou J, Hu Y, Li W, Zhi Q and Zhu X: Serum miR-372 is a diagnostic and prognostic biomarker in patients with early colorectal cancer. Anticancer Agents Med Chem 16: 424-431, 2016.

32. Wu G, Wang Y, Lu X, He H, Liu H, Meng X, Xia S, Zheng K and Liu B: Low miR-372 expression correlates with poor prognosis and tumor metastasis in hepatocellular carcinoma. BMC Cancer 15: 182, 2015

33. Kong X, Qian X, Duan L, Liu H, Zhu Y and Qi J: MicroRNA-372 suppresses migration and invasion by targeting p65 in human prostate cancer cells. DNA Cell Biol 35: 828-835, 2016.

34. Liu BL, Sun KX, Zong ZH, Chen S and Zhao Y: MicroRNA-372 inhibits endometrial carcinoma development by targeting the expression of the Ras homolog gene family member C (RhoC). Oncotarget 7: 6649-6664, 2016.

35. Ragusa M, Statello L, Maugeri M, Majorana A, Barbagallo D, Salito L, Sammito M, Santonocito M, Angelica R, Cavallaro A, et al: Specific alterations of the microRNA transcriptome and global network structure in colorectal cancer after treatment with MAPK/ERK inhibitors. J Mol Med (Berl) 90: 1421-1438, 2012.

36. Chen H, Zhang Z, Lu Y, Song K, Liu X, Xia F and Sun W: Downregulation of ULK1 by microRNA-372 inhibits the survival of human pancreatic adenocarcinoma cells. Cancer Sci 108: 1811-1819, 2017.

37. Tian RQ, Wang XH, Hou LJ, Jia WH, Yang Q, Li YX, Liu M, Li X and Tang H: MicroRNA-372 is down-regulated and targets cyclin-dependent kinase 2 (CDK2) and cyclin A1 in human cervical cancer, which may contribute to tumorigenesis. J Biol Chem 286: 25556-25563, 2011.

38. Ciró M, Prosperini E, Quarto M, Grazini U, Walfridsson J, McBlane F, Nucifero P,Pacchiana G, Capra M, Christensen J, et al: ATAD2 is a novel cofactor for MYC, overexpressed and amplified in aggressive tumors. Cancer Res 69: 8491-8498, 2009.

39. Hwang HW, Ha SY, Bang $\mathrm{H}$ and Park CK: ATAD2 as a poor prognostic marker for hepatocellular carcinoma after curative resection. Cancer Res Treat 47: 853-861, 2015.

40. Wan WN, Zhang YX, Wang XM, Liu YJ, Zhang YQ, Que YH and Zhao WJ: ATAD2 is highly expressed in ovarian carcinomas and indicates poor prognosis. Asian Pac J Cancer Prev 15: 2777-2783, 2014

41. Shang P, Meng F, Liu Y and Chen X: Overexpression of ANCCA/ ATAD2 in endometrial carcinoma and its correlation with tumor progression and poor prognosis. Tumour Biol 36: 4479-4485, 2015.

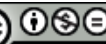

This work is licensed under a Creative Commons Attribution-NonCommercial-NoDerivatives 4.0 International (CC BY-NC-ND 4.0) License. 